\section{Two respiratory viruses, one antibody}

\section{By Tracey Baas, Senior Editor}

Humabs BioMed S.A. and the Institute for Research in Biomedicine at the University of Lugano have identified an antibody that could offer multiple advantages over the blockbuster respiratory syncytial virus prophylactic Synagis palivizumab. The group expects that their product, MPE8, could be used to both treat and prevent respiratory syncytial virus. It also could be effective against metapneumovirus, another common respiratory virus. ${ }^{1}$

Respiratory syncytial virus (RSV) and metapneumovirus (MPV) both can cause severe disease in premature newborns, hospitalized children and immunecompromised adults. ${ }^{2-5}$

Synagis from AstraZeneca plc's MedImmune LLC unit is marketed to prevent $\mathrm{RSV}$ infection in high-risk infants. The mAb posted sales of $\$ 1.04$ billion in 2012 but is not indicated as a therapeutic ${ }^{6}$ and has been shown
"MPE8 represents a nextgeneration palivizumab with higher potency on RSV and higher breadth, being able to neutralize MPV as well. This considerably extends the target population and the therapeutic indications."

-Alcide Barberis, Humabs BioMed S.A. neutralized a panel of RSV and MPV strains as well as two related animal paramyxoviruses. The antibody was about eightfold more potent than Synagis at neutralizing RSV. It also prevented RSV from spreading from cell to cell, suggesting MPE8 has potential as a therapeutic in patients in which infection already has taken hold.

In a mouse model of RSV infection, MPE8 given four hours before RSV nasal challenge decreased pulmonary infection 5- to 10-fold more than Synagis. In a mouse model of MPV infection, prophylactic use of MPE8 four hours before MPV nasal challenge decreased pulmonary infection compared with prophylactic use of vehicle.

The RSV mouse model is limited because it shows only modest viral replication, so the team next used a mouse model of infection with pneumonia virus of mice (PVM), which is lethal in mice and recapitulates the symptoms and pathology of human severe RSV infection. In the PVM mouse model, MPE8 given four hours before PVM nasal challenge protected the animals from lethality when given at doses as low as $0.12 \mathrm{mg} / \mathrm{Kg}$. Furthermore, MPE8 also protected the mice when given up to three days after PVM nasal challenge, whereas ribavirin was ineffective.

The authors did not report virus titer data and instead showed percent reductions in viral mRNA, which they quantified using RT-PCR. Showing log reductions of virus titers would have required plaque assays, which measure actual virus.

Results were published in Nature.

\section{Going viral}

Humabs is in discussions with companies to license MPE8 as well as other potent RSVin vivo to lead to the generation of viral escape mutants resistant to the drug.?

There is no targeted therapeutic for MPV infection. Treatment includes routine symptomatic care and respiratory support. Ribavirin, which has broad antiviral activity, is the only approved therapy for RSV and is sometimes used as a last-ditch treatment in children with severe infections by RSV or MPV.

Both viruses are members of the paramyxovirus family and share $33 \%$ amino acid identity in the F protein, which is the primary target of neutralizing antibodies. ${ }^{8,9}$

A Humabs-led team hypothesized that rare cross-neutralizing antibodies - those able to inhibit both RSV and MPV-might exist in the sera of individuals who have been repeatedly exposed to both viruses.

The group screened a cohort of 200 blood donors and selected 7 individuals who showed high levels of serum neutralizing antibodies against both viruses. The researchers then immortalized memory B cells isolated from the donors and determined if any antibodies produced by the B cells could neutralize both RSV and MPV.

The team identified 30 antibodies against RSV, and of those, 2 also had activity against MPV.

In vitro, one of the cross-reactive antibodies, dubbed MPE8, specific antibodies described in the study.

"MPE8 represents a next-generation palivizumab with higher potency on RSV and higher breadth, being able to neutralize MPV as well. This considerably extends the target population and the therapeutic indications," Humabs president and CEO Alcide Barberis told SciBX.

"We believe that MPE8 could represent a strong candidate for therapy of severe RSV/MPV infections," said Antonio Lanzavecchia, director of the Institute for Research in Biomedicine at the University of Lugano and a Humabs cofounder. "Proof of concept in humans could be obtained with a small trial in transplanted patients with documented RSV or MPV upper respiratory tract infection to block spread from upper to lower respiratory tract."

Julian Symons, senior director of product development at infectious disease company Alios BioPharma Inc., thought that tests of MPE8 in more traditional RSV and MPV animal models, which include the cotton rat and nonhuman primates, would be appropriate. mice," said Symons.

He added, "It would be interesting to assess how broadly neutralizing MPE8 is against the members of the paramyxovirus family. The paper describes neutralizing activity versus RSV, MPV, BRSV [bovine RSV] and PMV [paramyxovirus], which are all members of the Pneumovirinae
"Both viruses replicate more robustly in cotton rats than they do in 


“It would be interesting
to assess how broadly
neutralizing MPE8 is
against the members of
the paramyxovirus family.
The paper describes
neutralizing activity
versus RSV, MPV, BRSV
[bovine RSV] and PMV
[paramyxovirus], which
are all members of the
Pneumovirinae subfamily.
Does MPE8 neutralize
other paramyxoviruses
such as the
Paramyxovirinae subfamily
members mumps,
measles, hendra virus or
parainfluenza viruses?”
$\quad$-Julian Symons,
Alios BioPharma Inc.

subfamily. Does MPE8 neutralize other paramyxoviruses such as the Paramyxovirinae subfamily members mumps, measles, hendra virus or parainfluenza viruses?"

$\mathrm{Vu}$ Truong, founder and CSO of Aridis $\mathbf{P h}$ a r m a c utic a l s LLC, wanted to see a more detailed analysis comparing Synagis with MPE8 against RSV in potency and strain coverage. "The team presents their viral clearance data in terms of percent reduction of viral mRNA. However, more compelling data would be viral clearance presented in terms of log reduction of virus titers,"

Truong said. "To be considered more effective, you would want to see at least a log or more decrease in viral replication and possibly reactivity against Synagis-resistant strains."

Richard Hegele, chair of laboratory medicine and pathobiology at the University of Toronto, said that the team should run in vivo studies in different species that investigate escape virus mutants or the possibility of using two or more RSV antibodies that target different F protein epitopes.

"The failure to select MPE8 escape mutants in vitro gives us confidence that escape mutants are either extremely rare or may not exist," said Lanzavecchia. "On this ground we suggest that a single antibody may be sufficient."
On the safety side, Truong said, "It would be useful to initially test MPE8 against a panel of human tissues to ensure the lack of crossreactivity."

Lanzavecchia agreed. "This is a standard procedure in the clinical development of antibodies. Our preliminary data indicate that there is no evident cross-reactivity."

Humabs has filed for a patent covering MPE8 and the RSV-specific antibodies described in the study. The IP is available for licensing.

MedImmune declined requests for an interview.

Baas, T. SciBX 6(37); doi:10.1038/scibx.2013.1016

Published online Sept. 26, 2013

\section{REFERENCES}

1. Corti, D. et al. Nature; published online Aug. 18, 2013; doi:10.1038/nature12442

Contact: Antonio Lanzavecchia, Institute for Research in Biomedicine at the University of Lugano, Bellinzona, Switzerland e-mail: lanzavecchia@irb.usi.ch

Contact: Davide Corti, Humabs BioMed S.A., Bellinzona, Switzerland e-mail: davide.corti@humabs.ch

2. Hall, C.B. et al. N. Engl. J. Med. 360, 588-598 (2009)

3. Edwards, K.M. et al. N. Engl. J. Med. 368, 633-643 (2013)

4. Williams, J.V. et al. N. Engl. J. Med. 350, 443-450 (2004)

5. Falsey, A.R. et al. N. Engl. J. Med. 352, 1749-1759 (2005)

6. de Fontbrune, F.S. et al. Clin. Infect. Dis. 45, 1019-1024 (2007)

7. Adams, O. et al. Clin. Infect. Dis. 51, 185-188 (2010)

8. Collins, P.L. \& Karron, R.A. in Fields Virology 6th edn, Vol. 1 (eds Knipe, D.M. et al.) Ch. 38, 1086-1123 (Lippincott Williams \& Wilkins, 2013)

9. van den Hoogen, B.G. et al. Nat. Med. 7, 719-724 (2001)

\section{COMPANIES AND INSTITUTIONS MENTIONED}

AstraZeneca plc (LSE:AZN; NYSE:AZN), London, U.K.

Alios BioPharma Inc., South San Francisco, Calif.

Aridis Pharmaceuticals LLC, San Jose, Calif.

Humabs BioMed S.A., Bellizona, Switzerland Institute for Research in Biomedicine at the University of Lugano, Bellizona, Switzerland Medlmmune LLC, Gaithersburg, Md.

University of Toronto, Toronto, Ontario, Canada 